\title{
Willingness to Receive Periodic Health Examination Based on the Health Belief Model Among the Elderly in Rural China: A Cross-Sectional Study
}

\author{
Zhuo Zhang' \\ Ai-Tian Yin ${ }^{2}$ \\ Ying Bian' \\ 'State Key Laboratory of Quality \\ Research in Chinese Medicine, Institute \\ of Chinese Medical Sciences, University \\ of Macau, Taipa, Macau, People's Republic \\ of China; ${ }^{2}$ School of Health Care \\ Management, Cheeloo College of \\ Medicine, Shandong University, Jinan, \\ People's Republic of China
}

Purpose: This study aimed to explore factors affecting behavioral intention of receiving periodic health examinations (hereafter, BIE) among people aged 60 and over in rural China, namely, Shandong Province, using the extended health belief model (EHBM).

Patients and Methods: Participants were selected using stratified multi-stage random sampling. Three cities were selected based on economic level. Subsequently, three counties and three villages were selected from each sample city and county. Finally, 30 respondents were selected from each sample village. Face-to-face surveys were conducted using a structured questionnaire between March and September 2017. Multiple linear regression was conducted to investigate the association between BIE and eight dimensions of EHBM: perceived susceptibility, perceived severity, perceived benefits, perceived barriers, perceived self-efficacy, cues to action, health knowledge, and social support.

Results: Of the 509 rural respondents aged 60 years and older, the average score of behavioral intention was $4.43 \pm 0.80$. Multivariate linear regression analysis demonstrated poor BIE among participants who were men, were current smoker, were current drinker, were aged 70 years or over, had lower social support, and perceived lower self-efficacy, less benefits, and more barriers. Among them, barriers were found to have the strongest association with $\mathrm{BIE}\left(\mathrm{B}^{\prime}=-0.556 ; \mathrm{p}<0.001\right)$. Qualitative interviews revealed that reasons for not receiving periodic health examinations (PHE) included pain, cost, difficulty in finding a health care provider, time and scheduling, potential lack of trust in the physician, and value of the PHE.

Conclusion: This study highlighted the importance of psychological variables in the acceptance of PHE among the elderly in rural China and provided insights for further intervention designs targeting identified groups and performed by general practitioners. Addressing medical mistrust, strengthening, and enhancing one's social support network and health communication channels, such as bulletin boards, may serve to facilitate BIE.

Keywords: extended health belief model, periodic health examination, general practitioners, behavioral intention, elderly, rural China

\section{Introduction}

With increasing life expectancy and declining fertility due to China's unique 36year one-child policy, China is experiencing rapid aging at a rate that is significantly faster than most countries. ${ }^{1}$ The increasing trend of population aging led to a dramatic increase in health and economic burdens. ${ }^{2}$ In addition, older adults with chronic disabling conditions not only have a reduced life expectancy but also significantly impact the mental health and quality of life of their caregivers. ${ }^{3}$
Correspondence: Ying Bian Institute of Chinese Medical Sciences, University of Macau, Avenida da

Universidade, Room 2055, N22 Building, Taipa, Macau SAR, People's Republic of China

Tel +853-66548926

Email bianyingum@163.com 
This situation is considered alarming, as chronic diseases are generally preventable Through periodic health examinations (PHE), abnormal signs can be detected at an early stage, and correct diagnosis and effective treatment measures can be administered early. ${ }^{4}$

PHE is a primary approach for general practitioners to perform clinical preventive services in China and delaying or avoiding unwanted outcomes through an organized PHE for people aged 60 years and older is a critical public health concern in China. ${ }^{5}$ The Chinese government officially approved the health management technical protocol for elderly people based on the principles of equitable access and use of clinical preventive services. This protocol, which was implemented formally on April 1, 2016, aimed to address the care needs of people aged 60 years and older and improve their quality of life. The protocol emphasized that people aged 60 years and older should be given periodic health assessments, where personal health records are updated, and an appointment is made for the next check. Notably, a dedicated approach to PHE and assessment was highlighted as a key priority.

The development of chronic diseases generally involves five stages: exposure, acquisition, advancement/ progression, complications, and death/disability. ${ }^{6}$ PHE helps to detect the impact factors of sub-health status at stage one, such as being overweight, obesity, smoking, alcohol use, and high blood pressure. A timely change in lifestyle or simple drug intervention can eliminate risk factors and reduce or avoid the occurrence of diseases. The second stage is the acquisition of early disease due to exposure. At this stage, PHE helps recognize asymptomatic but insidiously progressive diseases and postpone the development of subsequent adverse outcomes. PHE has been documented to be cost-saving or cost-effective. ${ }^{7}$ A study from Japan found a strong inverse association between older people's medical expenditure and the use of PHE. ${ }^{8}$ Several community-based interventions involving health screening and primary care have found significant reductions in chronic diseases, such as stroke, ${ }^{9}$ ischemic heart disease, ${ }^{10}$ and chronic obstructive pulmonary disease. ${ }^{11}$ There is also convincing evidence that PHE is helpful in the early diagnosis of cancer and prevention of progression and potentially death. ${ }^{12}$

The PHE participation rate in China is low. ${ }^{13}$ Moreover, surveys and focus groups found that rural residents were less likely to access recommended clinical preventive services than urban populations. ${ }^{14}$ However, the total prevalence of individual chronic disease risk factors among rural residents was higher than that for urban residents. ${ }^{15}$ They tended to have more healthcare needs, while their underuse of primary care services was strikingly apparent compared to urban populations. ${ }^{16}$ Exploring the influencing factors of this utilization is crucial for PHE implementation.

Health promotion programs based on theoretical frameworks were found to be more effective than programs that did not use theory. The Health Belief Model emphasizes how a person's perceptions induce motivation and further produce behavior. ${ }^{17}$ To date, few studies examined psychosocial variables as a potentially influencing factor on BIE among people aged 60 years and older in rural China, underpinned by an extended health belief model (EHBM). Considering the utilization rate of PHE among the rural elderly population in China is extremely low while their health tends to be poor, analyzing factors that affect BIE is a vital health assessment approach for the aging Chinese society. Therefore, this study aimed to contribute timely direct policy suggestions. This study used representative data of Shandong Province to explore BIE systematically and clarify its influencing factors among elderly people in rural China, primarily based on EHBM in which factors were categorized into several groups.

\section{Patients and Methods Design, Setting, and Participants}

The target population of the present study was people in rural Shandong aged 60 years and older. The inclusion criteria were age of 60 years or older, permanent residence in Shandong, and ability to read and write Chinese. Utilizing stratified multi-stage sampling, a structured cross-sectional face-to-face questionnaire survey was conducted with 509 elderly people from 27 rural villages in 3 counties in Shandong Province between March to September 2017. The survey examined PHE behavior in the previous oneyear period. A sampling procedure was conducted, which contained county level, township level, and village level. First, all counties in Shandong Province were independently sorted in descending order by economic development ranking into 3 groups, and one county was selected at random from each group. Second, all townships in each of the sampled counties were divided into three groups based on their economic development level, and one township was selected from each group as a representative county $(3 \times 3=$ 9 townships selected). Third, all villages in each sampled 
township were grouped into three subgroups according to their regional economic status, and one village was randomly selected from each subgroup $(3 \times 3 \times 3=27$ villages $)$. Finally, 30 individuals were randomly selected from each of the 27 villages. Thus, 810 rural residents were enrolled in the study and 805 completed the questionnaires with a response rate of $99.4 \%$. In this study we only selected information from people aged 60 years and older for analysis. Among the respondents, 509 met the inclusion criteria. Additionally, 20 seniors were randomly obtained from the research subjects for qualitative research.

Using the formula $\mathrm{n}=\frac{\mathrm{u}_{a}^{2} \pi(1-\pi)}{\delta^{2}}$, in which $\pi$ referred to the percentage of elderly people in rural areas using PHE $(53.6 \%), \delta=0.1 \pi, \alpha=0.05, \mathrm{u}_{0.05}=1.96$, the minimum sample size was calculated as $\mathrm{n}=346$. Therefore, a sample size of 509 was sufficient.

\section{Pilot Study}

A pilot study was conducted before the commencement of the study in February 2017 among 30 rural individuals aged 60 years and older in order to test the comprehensibility of the questionnaire items and establish the reliability of the questionnaire. Amendments were made to unfamiliar phrases that required clarification. In addition, experts from the School of Health Care Management at Shandong University assisted in revising the questionnaire. The pilot survey demonstrated a PHE usage rate of $53.6 \%$.

\section{Data Collection}

Trained students from the School of Health Care Management at Shandong University were recruited to conduct individual face-to-face surveys in participants' homes. The questionnaire items and response choices were read to older participants in local languages in order to accommodate poor vision and other geriatric conditions. In addition, qualitative investigation was conducted using individual indepth interviews with 20 participants selected utilizing simple random sampling to explore reasons for low participation rate of PHE. To ensure data accuracy and completeness, the results were verified by quality controllers after finishing each questionnaire.

\section{Questionnaire}

Structured self-designed questionnaires were used to assess PHE intention and each dimension of EHBM. The EHBM was adopted as a conceptual framework to predict healthrelated behavior, explain it, and further develop behavior change interventions (Figure 1). The EHBM is based on a hypothesis regarding the likelihood that individuals will take some preventive action and focus on adherence to preventive health behaviors. It has been commonly applied to address public health problems for risk reduction, prevention, and community health promotion. ${ }^{18}$ Behavioral intentions of receiving PHE (hereafter, BIE) and their determinants were measured using the eight domains of EHBM. According to this theory, individuals adopt certain preventive behaviors when they perceive themselves as susceptible to a disease or health problem (perceived susceptibility), perceive severity of the disease (perceived severity), perceive benefits of adopting a health behavior (perceived benefits), believe that the benefits of the behavior outweigh the costs (perceived barriers), have confidence in their ability to perform the action successfully (perceived self-efficacy), have health knowledge and social support, and there are factors that promote individuals to adopt such behaviors (cues to action).

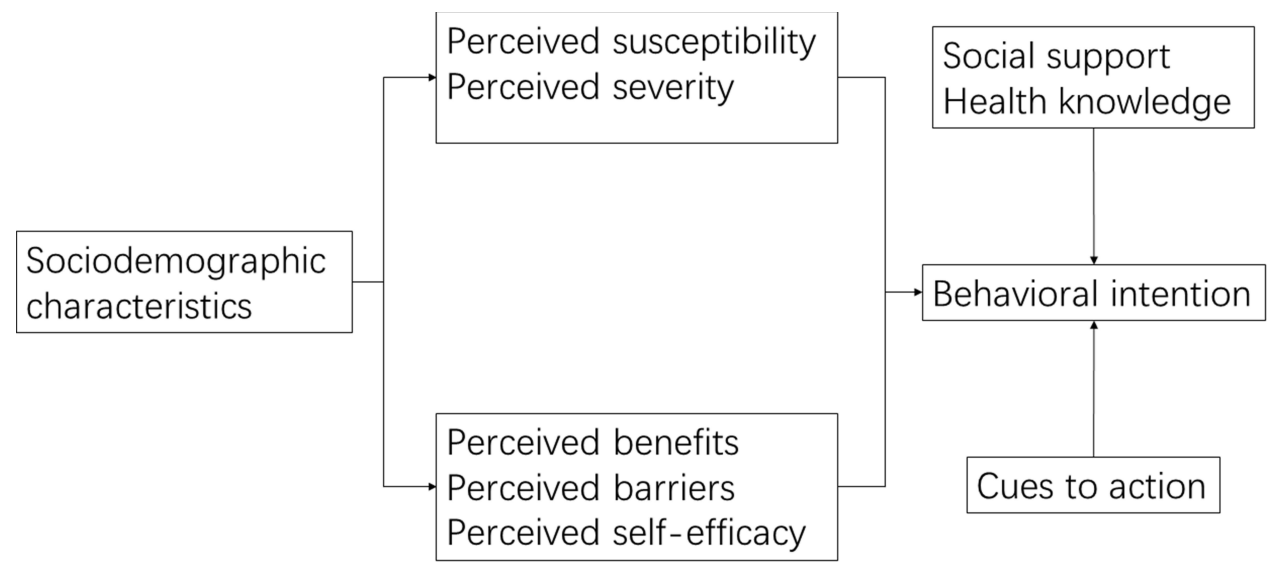

Figure I Conceptual framework based on extended health belief model. 
The questionnaire consisted of three parts. The first part included basic demographic information, such as sociodemographic data (gender, age, education level, marital status), economic status (per capita annual household income), health-related behavioral factor (tobacco use, alcohol use, exercise), and the need factor (number of noncommunicable chronic diseases). The second part was designed to explore BIE (1 item). The third part (49 items) targeted psychology, which was the major part based on selfdesigned eight-dimensional EHBM (Table 1). It contained statements regarding attitude and perceptions relating to PHE use behavior: perceived barriers ( 6 items), perceived benefits ( 3 items), perceived susceptibility ( 5 items), perceived severity (6 items), cues to action (5 items), self-efficacy (5 items), health knowledge (7 items), and social support (12 items). BIE, perceived barriers, perceived benefits, perceived susceptibility, perceived severity, cues to action, and selfefficacy were defined as an integrated view and these measurement items were revised from previous surveys. ${ }^{19-21}$ Furthermore, social support items were selected based previous research, ${ }^{22}$ which contained emotional support, tangible support, affectionate support, and positive interaction.

Table I Examples of the Questionnaire Items

\begin{tabular}{|l|l|l|l|}
\hline Variables & Items & Range & Item Example \\
\hline BIE & I & I 5 & $\begin{array}{l}\text { I intend to perform PHE } \\
\text { regularly. }\end{array}$ \\
\hline Self-efficacy & 5 & $5 \sim 25$ & $\begin{array}{l}\text { In general, I can exercise } \\
\text { regularly. }\end{array}$ \\
\hline $\begin{array}{l}\text { Perceived } \\
\text { susceptibility }\end{array}$ & 5 & $5 \sim 25$ & $\begin{array}{l}\text { I worry a lot about becoming ill if } \\
\text { I do not perform PHE. }\end{array}$ \\
\hline $\begin{array}{l}\text { Perceived } \\
\text { severity }\end{array}$ & 6 & $6 \sim 30$ & $\begin{array}{l}\text { If I had an illness, my career } \\
\text { would be endangered. }\end{array}$ \\
\hline $\begin{array}{l}\text { Perceived } \\
\text { benefits }\end{array}$ & 3 & $3 \sim 15$ & $\begin{array}{l}\text { When I perform PHE, I do not } \\
\text { worry as much about illnesses. }\end{array}$ \\
\hline $\begin{array}{l}\text { Perceived } \\
\text { barriers }\end{array}$ & 6 & $6 \sim 30$ & $\begin{array}{l}\text { Performing PHE can be time- } \\
\text { consuming. }\end{array}$ \\
\hline $\begin{array}{l}\text { Cues to } \\
\text { action }\end{array}$ & 5 & $5 \sim 25$ & $\begin{array}{l}\text { Village doctors' } \\
\text { recommendations prompted me } \\
\text { to perform PHE. }\end{array}$ \\
\hline $\begin{array}{l}\text { Health } \\
\text { knowledge } \\
\text { support }\end{array}$ & 7 & $7 \sim 35$ & $\begin{array}{l}\text { I am familiar with the clinical } \\
\text { preventive services. }\end{array}$ \\
\hline $\begin{array}{l}\text { Sobreval } \\
\text { I2 } 60\end{array}$ & $\begin{array}{l}\text { I can get the necessary emotional } \\
\text { support from my family. }\end{array}$ \\
\hline
\end{tabular}

Abbreviations: BIE, behavioral intention of PHE; PHE, periodic health examination.
Health knowledge items were modified based on a previous study. ${ }^{23}$ The respondents were asked to indicate their agreement with statements on a five-point Likert-type scale (strongly disagree $=1$, disagree $=2$, neutral $=3$, agree $=4$, strongly agree $=5$ ).

The Cronbach's $\alpha$ values for all the scales ranged from 0.72 to 0.94 . A panel of five experts, including family physicians, practitioner nurses, and public health experts, were invited to validate the questionnaire. Most items were evaluated by the experts to ensure appropriateness, with the Content Validity Index of 0.912 .

\section{Statistics}

Data was processed using Microsoft Access and analyzed using STATA 15.1. The characteristics of subjects were described with number (percent). Mean (SD) was used to describe the scores of EHBM and BIE, and t-tests or the oneway analysis of variance (ANOVA) were performed for groups comparisons. Pearson's correlation analysis was conducted between EHBM constructs and BIE. In addition, a multiple linear regression analysis was used to identify the association between EHBM and BIE, with potential confounding factors adjusted. Standardized regression coefficient (B') was used to explain the strength of the association. All $P$ values reported were two-sided, and $P$ values below 0.05 were considered statistically significant.

\section{Results}

\section{Participant Characteristics}

Table 2 presents participant characteristics. The participants were predominantly male $(65.6 \%)$ and had an average age of $69.3 \pm 7.1$ years. The majority $(78.2 \%)$ had completed no more than junior school education. Approximately half of the families (56.6\%) reported a per capita annual household income of less than 20,000 CNY (approximately 2950 USD). In addition, $75.6 \%$ of participants were married. Overall, 372 of the 509 respondents $(73.1 \%)$ had one or more chronic diseases. Furthermore, health-related behavior information was collected, including tobacco use, alcohol use, and regular exercise. Overall, $34.4 \%$ of the respondents reported currently being a smoker, $37.5 \%$ reported currently being a drinker, and $66.8 \%$ reported regular exercise.

\section{Scores of EHBM Variables}

Table 3 shows the scores of the participants' perceptions of PHE, and variables among respondents with different ages, 
Table 2 Characteristics of Rural Elderly Aged 60 Years and Over

\begin{tabular}{|c|c|c|}
\hline Characteristics & $\begin{array}{l}\text { Frequency } \\
(n=509)\end{array}$ & $\begin{array}{l}\text { Percentage } \\
\text { (\%) }\end{array}$ \\
\hline \multicolumn{3}{|c|}{ Socio-demographic characteristics } \\
\hline \multicolumn{3}{|l|}{ Age } \\
\hline$<70$ & 298 & 58.5 \\
\hline $70 \sim 80$ & 152 & 29.9 \\
\hline$\geq 80$ & 59 & 11.6 \\
\hline \multicolumn{3}{|l|}{ Gender } \\
\hline Male & 334 & 65.6 \\
\hline Female & 175 & 34.3 \\
\hline \multicolumn{3}{|l|}{ Marital status } \\
\hline Single & 124 & 24.4 \\
\hline Married & 385 & 75.6 \\
\hline \multicolumn{3}{|l|}{ Education level } \\
\hline Primary school or below & 398 & 78.2 \\
\hline Junior school or above & 111 & 21.8 \\
\hline \multicolumn{3}{|l|}{ Economic status } \\
\hline \multicolumn{3}{|c|}{ Per capita annual household } \\
\hline \multicolumn{3}{|c|}{ Income (yuan) } \\
\hline$<10,000$ & 187 & 36.7 \\
\hline $10,000 \sim 20,000$ & 101 & 19.8 \\
\hline$\geq 20,000$ & 221 & 43.4 \\
\hline \multicolumn{3}{|c|}{ Health-related behavioral factor } \\
\hline \multicolumn{3}{|l|}{ Tobacco use } \\
\hline Nonsmoker & 263 & 51.7 \\
\hline Ex-smoker & 71 & 13.9 \\
\hline Current smoker & 175 & 34.4 \\
\hline \multicolumn{3}{|l|}{ Alcohol use } \\
\hline Nondrinker & 258 & 50.7 \\
\hline Abstainer & 60 & 11.8 \\
\hline Current drinker & 191 & 37.5 \\
\hline \multicolumn{3}{|l|}{ Exercise regularly } \\
\hline No & 169 & 33.2 \\
\hline$<45 \mathrm{~min} / \mathrm{d}$ & 182 & 35.8 \\
\hline$\geq 45 \mathrm{~min} / \mathrm{d}$ & 158 & 31.0 \\
\hline \multicolumn{3}{|l|}{ Need factor } \\
\hline \multicolumn{3}{|l|}{ Chronic disease status } \\
\hline No & 137 & 26.9 \\
\hline Yes & 372 & 73.1 \\
\hline
\end{tabular}

gender, education levels, per capita annual household income, marital status, health-related behavioral factors, and chronic disease status. Of the 509 respondents, the average score of behavioral intention was 4.43 ( 0.80 : behavioral intention), and scores of the eight dimensions of
EHBM were 21.33 (3.18: self-efficacy), 21.32 (5.51: health knowledge), 46.72 (13.08: social support), 17.87 (5.12: perceived susceptibility), 11.22 (3.68: perceived severity), 12.12 (2.90: perceived benefits), 10.91 (5.28: perceived barriers), 19.83 (4.73: cues to action), respectively.

As depicted in Table 3, participants who had higher BIE were more likely to be 60-69 years old, female, exsmoker, abstainer, had per capita annual household income ranging from 10,000 to 20,000 CNY (1473-2950 USD). Participants who were male $(\mathrm{P}<0.05)$, were ex-smoker $(\mathrm{P}<0.05)$, were abstainer $(\mathrm{P}<0.05)$ and exercised regularly $(\mathrm{P}<0.05)$ perceived more self-efficacy. Participants who were aged 60-69 $(\mathrm{P}<0.05)$, were ex-smoker $(\mathrm{P}<0.05)$ and were abstainer $(\mathrm{P}<0.05)$ perceived more social support. Participants who were female $(\mathrm{P}<0.05)$, were ex-smoker $(\mathrm{P}<0.05)$, were abstainer $(\mathrm{P}<0.05)$ and exercised regularly $(\mathrm{P}<0.05)$ perceived more susceptibility. Participants who had lower education level $(\mathrm{P}<0.05)$, were female $(\mathrm{P}<0.05)$, and had no habit of smoking and drinking $(\mathrm{P}<0.05)$ perceived more severity. Participants who were aged 60-69 $(\mathrm{P}<0.05)$, were female $(\mathrm{P}<0.05)$, were ex-smoker $(\mathrm{P}<0.05)$, were abstainer $(\mathrm{P}<0.05)$ and had an per capita annual household income ranging from 10,000 to 20,000 CNY (1473-2950 USD; $\mathrm{P}<0.05)$ perceived more benefits. Participants who were aged $\geq 80 \quad(\mathrm{P}<0.05)$, were male $(\mathrm{P}<0.05)$, had per capita annual household income below $10,000 \mathrm{CNY}$ (1473 USD; $\mathrm{P}<0.05)$, and had a habit of smoking $(\mathrm{P}<0.05)$ and drinking $(\mathrm{P}<0.05)$ perceived more barriers. Participants who were aged 60-69 $(\mathrm{P}<0.05)$ and had an per capita annual household income ranging from $10,000$ to 20,000 CNY (1473-2950 USD; $\mathrm{P}<0.05)$ scored higher on cues to action.

\section{Pearson's Correlation Analysis Between BIE and EHBM Constructs}

Table 4 shows the correlations between each of the EHBM construct and BIE using Pearson's correlation analysis. The results revealed that BIE was significantly correlated with self-efficacy, social support, susceptibility, benefits, barriers, and cues to action. $\mathrm{P}<0.05$ was considered statistically significant. Positive correlations were found between BIE and perceived self-efficacy, social support, perceived susceptibility, perceived benefits, and cues to action. Negative correlations were revealed between BIE and perceived barriers. BIE was not associated with health knowledge or perceived severity. 


\begin{tabular}{|c|c|c|c|c|c|c|c|c|c|c|c|c|c|c|c|c|}
\hline \multirow{2}{*}{ 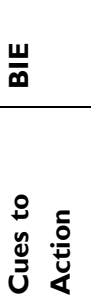 } & \multirow{2}{*}{ 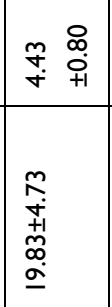 } & \multicolumn{3}{|c|}{ 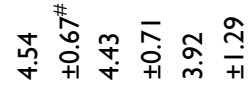 } & \multicolumn{2}{|c|}{ 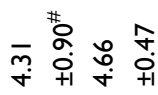 } & \multicolumn{2}{|c|}{ 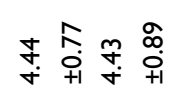 } & \multicolumn{3}{|c|}{ 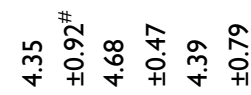 } & \multicolumn{2}{|c|}{ 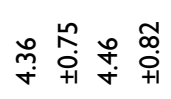 } & \multicolumn{3}{|c|}{ 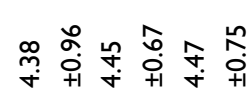 } \\
\hline & & 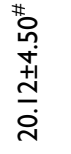 & $\begin{array}{l}\stackrel{2}{m} \\
\stackrel{+}{+1} \\
\sigma \\
\stackrel{\sigma}{\sigma}\end{array}$ & 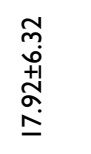 & $\begin{array}{l}0 \\
0 \\
\dot{0} \\
+1 \\
0 \\
0 \\
0 \\
\underline{0}\end{array}$ & $\begin{array}{l}\frac{0}{\dot{+}} \\
\frac{+1}{ \pm} \\
\frac{i}{\dot{d}}\end{array}$ & 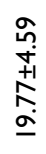 & 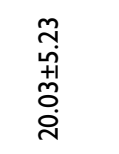 & 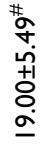 & 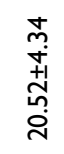 & $\begin{array}{l}\frac{0}{\dot{+}} \\
\frac{+1}{N} \\
\stackrel{N}{0}\end{array}$ & 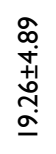 & 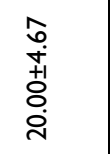 & 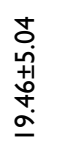 & 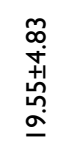 & $\begin{array}{l}\bar{N} \\
+ \\
+1 \\
\tilde{N} \\
\stackrel{N}{0}\end{array}$ \\
\hline صَ & 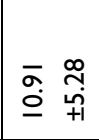 & \multicolumn{3}{|c|}{ 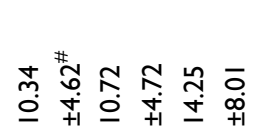 } & \multicolumn{2}{|c|}{ 菖 } & \multicolumn{2}{|c|}{ 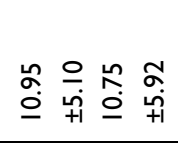 } & \multicolumn{3}{|c|}{ 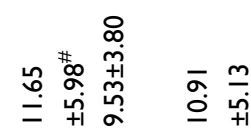 } & \multicolumn{2}{|c|}{ 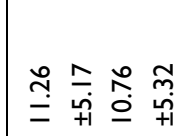 } & \multicolumn{3}{|c|}{ 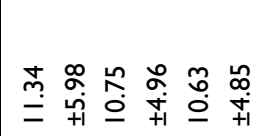 } \\
\hline ڤે & 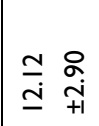 & \multicolumn{3}{|c|}{ 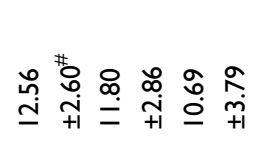 } & \multicolumn{2}{|c|}{ 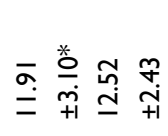 } & \multicolumn{2}{|c|}{ 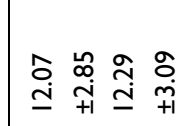 } & \multicolumn{3}{|c|}{ 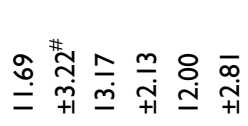 } & \multicolumn{2}{|c|}{ 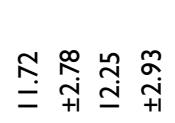 } & \multicolumn{3}{|c|}{ 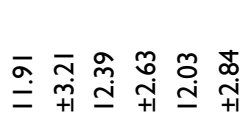 } \\
\hline 髉 & 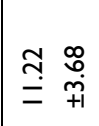 & \multicolumn{3}{|c|}{ 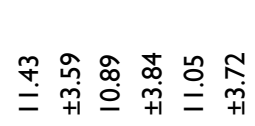 } & \multicolumn{2}{|c|}{ 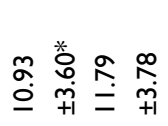 } & \multicolumn{2}{|c|}{ 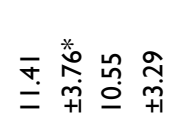 } & \multicolumn{3}{|c|}{ 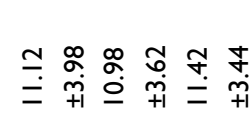 } & \multicolumn{2}{|c|}{ 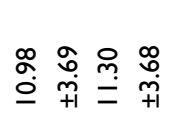 } & \multicolumn{3}{|c|}{ 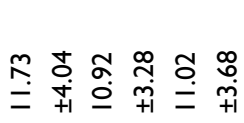 } \\
\hline 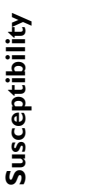 & 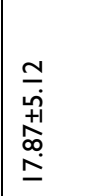 & $\begin{array}{l}\infty \\
\infty \\
+ \\
+ \\
\pm \\
\infty \\
\underline{\infty}\end{array}$ & 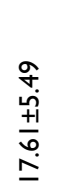 & 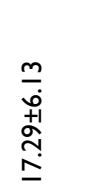 & & 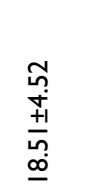 & & 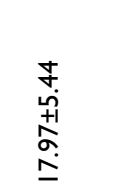 & & 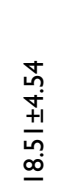 & 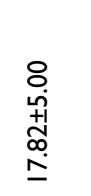 & $\begin{array}{l}\infty \\
0 \\
i+1 \\
+1 \\
\stackrel{0}{0} \\
\underline{\Sigma}\end{array}$ & $\begin{array}{l}\frac{t}{\dot{d}} \\
\frac{+1}{a} \\
\stackrel{+}{\Lambda}\end{array}$ & & 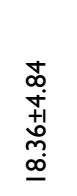 & $\begin{array}{l}\bar{\infty} \\
\stackrel{+}{+1} \\
\underset{\sim}{+1} \\
\underline{\infty}\end{array}$ \\
\hline 焉 & 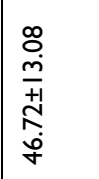 & 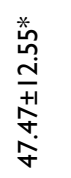 & 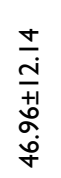 & 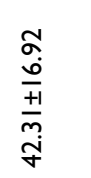 & & $\begin{array}{l}\hat{̃} \\
\overline{\overline{+1}} \\
\frac{0}{\infty} \\
\frac{\infty}{f}\end{array}$ & 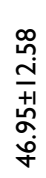 & 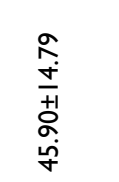 & & $\begin{array}{l}\overline{\bar{i}} \\
\bar{i} \\
\stackrel{+}{0} \\
\dot{\alpha}\end{array}$ & $\begin{array}{l}\bar{i} \\
\bar{i} \\
+\frac{j}{j} \\
\dot{j}\end{array}$ & 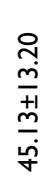 & $\begin{array}{l}\tilde{O} \\
\stackrel{\dot{m}}{+1} \\
\stackrel{\tilde{N}}{\tilde{q}}\end{array}$ & 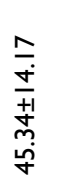 & 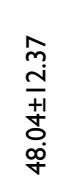 & 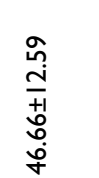 \\
\hline 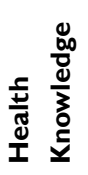 & 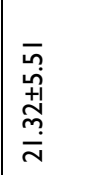 & 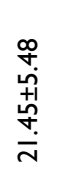 & $\begin{array}{l}\hat{f} \\
\dot{\omega} \\
+1 \\
\stackrel{+}{0} \\
\dot{n}\end{array}$ & 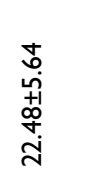 & & 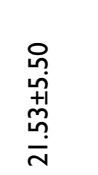 & 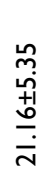 & 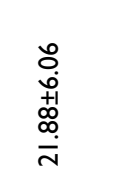 & 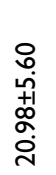 & 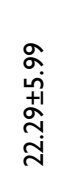 & 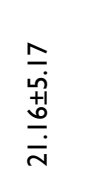 & 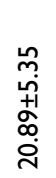 & 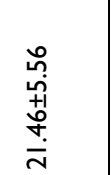 & & $\begin{array}{l}\bar{a} \\
\dot{p} \\
\dot{+1} \\
\dot{m} \\
\bar{N}\end{array}$ & 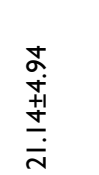 \\
\hline 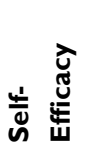 & $\begin{array}{l}\frac{\infty}{N} \\
\stackrel{m}{m} \\
\stackrel{m}{N} \\
\bar{N}\end{array}$ & 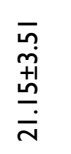 & 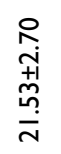 & 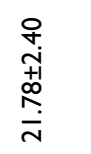 & $\frac{n}{\text { ํ. }}$ & & & 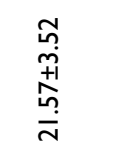 & & 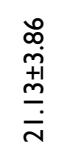 & $\begin{array}{l}\stackrel{t}{0} \\
\stackrel{+}{+} \\
\stackrel{+}{\oplus} \\
\stackrel{N}{N}\end{array}$ & $\begin{array}{l}\stackrel{\infty}{\infty} \\
\stackrel{+}{+} \\
\stackrel{+}{+} \\
\stackrel{N}{N}\end{array}$ & $\begin{array}{l}\stackrel{\infty}{\sim} \\
\stackrel{+}{+1} \\
\frac{m}{n}\end{array}$ & & 辛 & 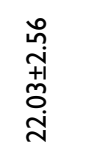 \\
\hline & & $\stackrel{?}{\mathrm{v}}$ & $\begin{array}{l}\infty \\
\infty \\
\vdots \\
1 \\
1\end{array}$ & $\stackrel{\circ}{\circ}$ & & 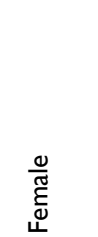 & & 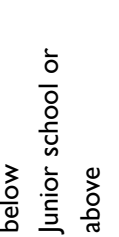 & $\begin{array}{l}8 \\
\frac{0}{v}\end{array}$ & 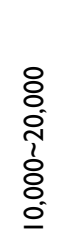 & 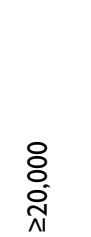 & $\begin{array}{l}\frac{0}{00} \\
\text { in }\end{array}$ & 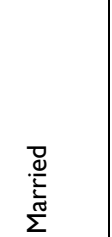 & $\stackrel{\circ}{z}$ & 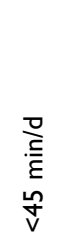 & 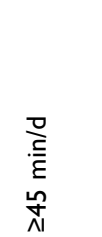 \\
\hline 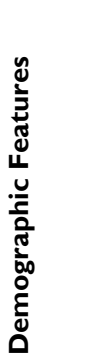 & 䓞 & 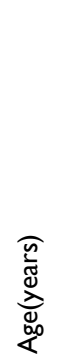 & & & 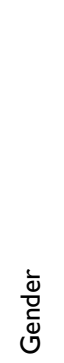 & & 홈 & & 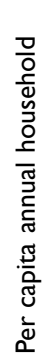 & & & 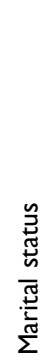 & & 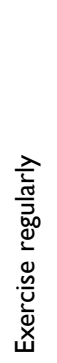 & & \\
\hline
\end{tabular}




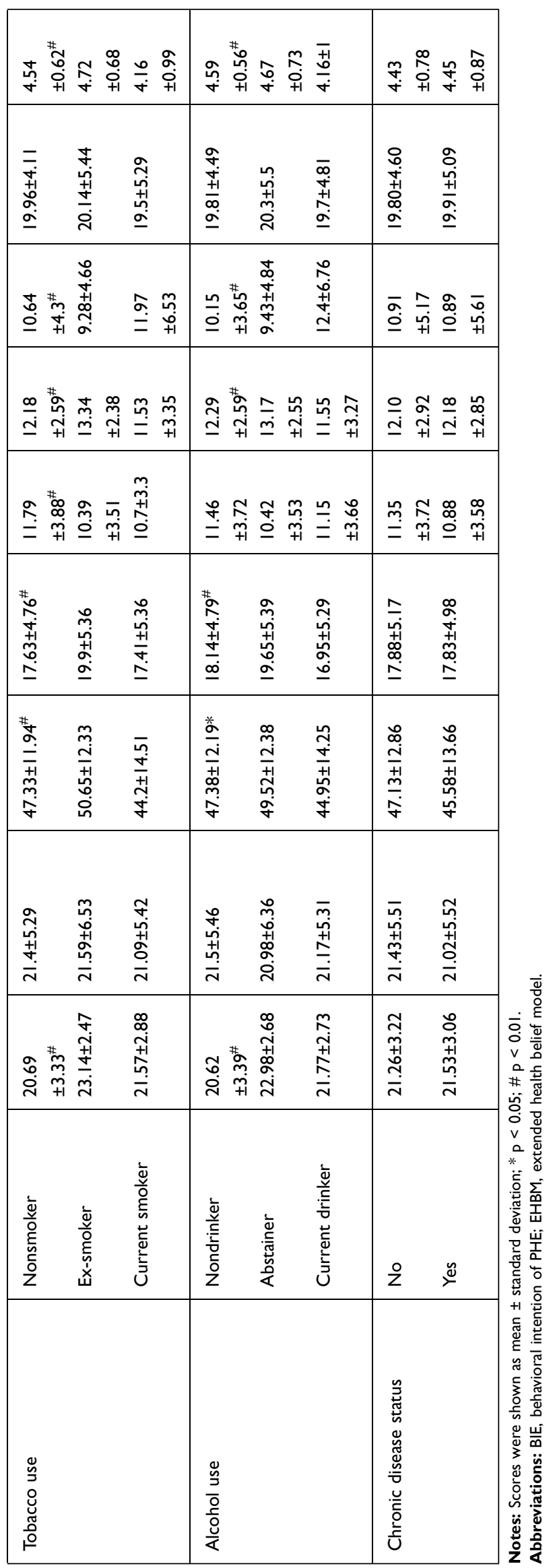


Table 4 Pearson's Correlation Analysis Between BIE and EHBM Constructs

\begin{tabular}{|l|l|l|l|l|l|l|l|l|}
\hline & Self-Efficacy & Health Knowledge & Social Support & Susceptibility & Severity & Benefits & Barriers & Cues to Action \\
\hline$r$ & 0.252 & -0.012 & 0.485 & 0.392 & -0.046 & 0.650 & -0.800 & 0.448 \\
$P$ & 0.000 & 0.779 & 0.000 & 0.000 & 0.298 & 0.000 & 0.000 & 0.000 \\
\hline
\end{tabular}

\section{Predictors Affecting BIE Among People Aged 60 or Over in Rural Shandong}

A multiple linear regression analysis was implemented to adjust for the effects of other confounding variables affecting BIE among the participants. All variables, including EHBM constructs as well as the demographic characteristics of the respondents, were entered as independent variables to predict the dependent variable, BIE.

As shown in Table 5, eight predicting variables, including age, gender, tobacco use, alcohol use, self-efficacy, social support, benefits, and barriers, were retained in the multiple linear regression analysis model to predict BIE (Table 5). Among all the significant predictors, the factor of perceived barriers had the highest standardized regression coefficient $\left(B^{\prime}=-0.556 ; p<0.001\right)$. That is, participants who perceived fewer barriers were more likely to have a higher BIE. Moreover, benefits, self-efficacy, and social support demonstrated relative higher contributions toward BIE, suggesting that significant variables in the extended health belief model were important in predicting BIE. With an $\mathrm{R}^{2}$ of $64.2 \%$, the overall variance in BIE accounted for by the model appeared

Table 5 Multivariate Linear Regression Analysis Model Examining Predictors Associated with BIE Among Rural Elderly Aged 60 or Over in Shandong

\begin{tabular}{|l|l|l|l|l|l|}
\hline Predictor & B & B' $^{\prime}$ & SE & T & P \\
\hline Constant & 3.415 & & 0.308 & $I I .070$ & 0.000 \\
Age & -0.076 & -0.066 & 0.030 & -2.540 & 0.011 \\
Gender & 0.137 & 0.081 & 0.053 & 2.560 & 0.011 \\
\hline Tobacco use & & & & & \\
$\quad$ Ex-smoker & -0.026 & -0.011 & 0.100 & -0.260 & 0.796 \\
$\quad$ Current smoker & -0.162 & -0.097 & 0.049 & -3.300 & 0.001 \\
\hline Alcohol use & & & & & \\
$\quad$ Abstainer & -0.086 & -0.035 & 0.101 & -0.850 & 0.393 \\
Current drinker & -0.105 & -0.063 & 0.048 & -2.200 & 0.028 \\
\hline Self-efficacy & 0.034 & 0.136 & 0.007 & 4.840 & 0.000 \\
Social support & 0.003 & 0.057 & 0.002 & 2.050 & 0.041 \\
Benefits & 0.069 & 0.249 & 0.008 & 8.540 & 0.000 \\
Barriers & -0.084 & -0.556 & 0.005 & -15.870 & 0.000 \\
\hline
\end{tabular}

Notes: $R^{2}=0.642$. $B^{\prime}$ :standardized regression coefficient. to be high. Thus, the model had a good prediction for BIE. However, severity, susceptibility, cues to action, and health knowledge subscales did not show a relation to BIE.

\section{Discussion}

The present study revealed significant potential for improvement in BIE among the participants. This study focused on EHBM-based psychological variables, as these psychological variables could be targeted for further interventions to change the low participation rate in PHE. This study utilized behavioral intention as an outcome variable to determine the effect of EHBM dimensions on BIE. The explanatory power of the six-dimensional HBM is limited in predicting health behaviors, ${ }^{24-26}$ particularly in some specific studies. ${ }^{27}$ This study added two dimensions to the HBM model, namely, health knowledge and social support, to construct an eight-dimensional EHBM in order to improve the predictive validity. Health knowledge is believed to promote attitude change before behavior change occurs. ${ }^{28}$ In addition, Chinese older adults who live alone or lack social support have extremely low utilization of preventive health services, ${ }^{29}$ and rural Chinese older populations face severe social support deficits due to rural-urban migration. Social support can significantly influence physical and mental health status of older adults. ${ }^{30}$ Therefore, the variable of social support was added to the HBM.

Consistent with the model's predictions, participants were more likely to engage in PHE services if they had high self-efficacy, few perceived barriers, high-perceived benefits, and received more social support. Of these, perceived barriers were the most important determinant, followed by perceived benefits, self-efficacy, and social support. Past HBM-based meta-analyses and reviews of critical quantitative studies have shown that important predictors of behavior were typically perceived susceptibility, perceived severity, perceived barriers, and perceived benefits. ${ }^{31-33}$ This study found that perceived benefits were independently associated with performing PHE, which was consistent with Rosenstock and Alhalaseh. ${ }^{34,35}$ Likewise, a recent study showed that 
perceived barriers and perceived benefits were the two strongest predictors of individual intention to receive the COVID-19 vaccine. ${ }^{36}$ An article exploring predictors of condom use intention among female sex workers in two cities in Hubei Province, China, showed that perceived benefits and barriers were the most direct determinants of condom use. ${ }^{37}$ Therefore, there is a need to focus on increasing awareness of PHE benefits while reducing the identified barriers.

Previous studies found that high self-efficacy had a positive effect on intention to preventive health behaviors. Zhao confirmed that higher self-efficacy was associated with more positive perceptions of condom use. ${ }^{37}$ In addition, scholars have noted that higher self-efficacy was a predictor of improved alcohol treatment outcomes. ${ }^{38}$ This study is in line with previous studies that cited the importance of self-efficacy in BIE in older adults, ${ }^{39}$ and demonstrated that people who were more confident and competent in obtaining PHE were more likely to do it regularly. ${ }^{39}$ Interventions based on self-efficacy theory are also thought to help promote health behavior change. ${ }^{40}$

Based on existing studies documenting that social support has a positive effect on BIE in different populations, ${ }^{41-43}$ this study found that participants with more social support were more likely to report scheduling PHE. Mitsuhashi showed that older adults who received PHE had friends who were members of various groups, ${ }^{43}$ such as neighborhood associations and clubs. Another survey demonstrated that social support was a significant predictor of annual use of physical health screening. ${ }^{42}$ These results provide direct evidence indicating the irreplaceable role of social support in effectively reducing feelings of anxiety, improving health perceptions, and maintaining a healthy physical and psychological state, which in turn contribute to positive PHE acceptance. In addition, the present study confirmed these findings and further suggested the inclusion of social support into interventions to increase PHE acceptance.

Furthermore, this study suggested that health knowledge, perceived severity, perceived susceptibility, and cues to action did not predict BIE. A meta-analysis study of willingness to screen for cancer showed that only cues to action, health literacy, and perceived susceptibility positively predicted willingness. ${ }^{44}$ Another investigation showed that cues to action, perceived severity, and perceived benefits had a significant impact on women's choice of mode of birth. ${ }^{45}$ A survey in western Turkey showed that knowledge was a significantly associated variable with preventive health screening for breast cancer. ${ }^{46}$ However, this association was not maintained in the relationship between knowledge, gender, and health service use in Green's household interview survey. ${ }^{47}$ Similarly, several other studies found that perceived severity was consistently not an important predictor of preventive health behaviors. ${ }^{48,49}$ This may be related to the chosen focus groups. In the present study, respondents did not have a specific illness that required urgent care. Therefore, they did not perceive severity. Additionally, perceived susceptibility failed to explain the acceptance of PHE services among the participants, which was consistent with Carpenter. ${ }^{31}$ This difference could be explained by the fact that the events studied were behavioral intentions during major disease screenings, such as cancer screening, or emergency time decisions, such as childbirth. When people are faced with these events, they require advice from medical professionals and expertise to help them, and they can directly feel the susceptibility and severity of the threat of the disease. In addition, $94.3 \%$ of the participants had an education level of junior high school or less. Thus, their education level could not influence their behavioral intentions. Among elderly people in rural areas of China, PHE may be perceived as an unnecessary event, which to some extent affects their perception of the severity of the disease. Further, older people in rural areas may not believe in the value of preventive health services compared to clinical treatments.

The findings on the factors that influence BIE suggested the importance of targeting specific characteristics of non-users, including gender, age, tobacco use, and alcohol use. These may provide information necessary to promote the use of PHE in these individuals. For instance, as predicted by EHBM, men had weaker BIE. Many scholars reported that women experience more illness, are more sensitive to their health status, and have poor self-reported health status. As a result, they use preventive health services more than men do. ${ }^{50}$ However, men are less willing to take on the role of patient and receive preventive health services, as traditional men fear revealing their weaknesses. ${ }^{51}$ In addition, researchers found that women had more health knowledge than men did, which is thought to account for higher health-seeking behaviors. As a result, men had lower behavioral intentions to use PHE and seek medical help. ${ }^{52}$

Based on qualitative interviews with participants, difficulty in finding a provider, time and scheduling, possible lack of trust in physicians, pain, cost, and beliefs about the 
value of PHE were the main barriers to PHE among the participants. A qualitative study of Korean American women found that the main barriers were cost, time, language, fear, denial, and Confucianism, ${ }^{53}$ which supports the present results. In addition, distance to the nearest medical facility was a barrier, particularly for participants with physical mobility problems. Low trust in doctors and low confidence in PHE may be due to a lack of effective health communication channels for elderly people in rural areas. Health facilities must improve the health literacy of older adults in rural areas to increase willingness to participate in PHE. Health communication provided by family physicians may be an effective strategy to improve health literacy and change attitudes and beliefs about preventive health services, further promoting the use of PHE. In addition, watching television was found to be another effective way to improve health literacy. A survey in western Turkey reported that nearly $40 \%$ of the study group received key information about breast cancer screening from television. ${ }^{46}$ Furthermore, bulletin boards in rural China are an effective health communication channel due to their credibility as representatives of the public and the government.

\section{Strengths and Limitations}

To our knowledge, there is no EHBM applicable to Chinese rural elderly and comprehensively to general PHE as opposed to specific titles. The data presented in this study offered the perceptions of elderly people in rural China regarding $\mathrm{PHE}$ and determinants of PHE acceptance, which may provide a reference for the health agency. This sample may have differed from others in this issue in that it did not focus on urban people. Furthermore, the participants reported diverse demographic backgrounds, which likely affected the psychological variables and use of PHE. One of the limitations to this study is that some predictors found by other researchers were not included. Another research limitation is that this study used closed-ended questions, which may have allowed participants to guess the correct answer. In addition, this study was cross-sectional, and causality could not be assumed. Moreover, as data were self-reported, there is a risk of self-report bias.

\section{Conclusion}

The present study highlighted a need to perform health interventions for people aged 60 years and older, particularly in rural areas. Health interventions are required especially on targeted individuals who perceive more barriers, less self-efficacy, less benefits, and had less social support as well as those who are men, aged 70 years and over, with smoking or drinking behavior. As physicians largely determine the utilization of existing services, general practitioners should be encouraged to screen all elderly people regarding various risk factors and, in addition to appropriate intervention, consider reminding people who are at higher risk of the PHE repeatedly when their PHE is due. Other health communication channels, such as bulletin boards and television based in the community, should be used frequently to help elderly people in rural areas form a habit of using preventive health services. In the future, similar studies implementing in a wider range of areas should be considered to obtain a more reliable conclusion.

\section{Ethics Approval and Consent to Participate}

This study was approved by the Medical Ethics Committee of Medical School, Shandong University (LL201,401,048), and conforms to the ethics guidelines of the Declaration of Helsinki. Informed consent was obtained from all participants prior to questionnaire administration.

\section{Acknowledgment}

We would like to thank all participants in the study, all the staffs who coordinate the field work and all the investigators who contributed to data collection. This research was supported by the National Natural Science Foundation of China (71373147) and the research fund to YB from the University of Macau (MYRG2019-00044-ICMS). The funding bodies had no role in the study design, data collection and analysis, or the writing of the manuscript.

\section{Disclosure}

The authors report no conflicts of interest in this work.

\section{References}

1. Nan Y, Feng T, Hu Y, Qi X. Understanding Aging Policies in China: a Bibliometric Analysis of Policy Documents, 1978-2019. Int J Environ Res Public Health. 2020;17(16):5956. doi:10.3390/ ijerph17165956

2. Atella V, Piano Mortari A, Kopinska J, et al. Trends in age-related disease burden and healthcare utilization. Aging Cell. 2019;18(1): e12861. doi:10.1111/acel.12861

3. Liu J, Lu N, Lou VWQ. Care Tasks in the Stress Process for Family Caregivers in Urban China. Clin Gerontol. 2017;40(5):428-434. doi:10.1080/07317115.2017.1305033 
4. Hoang PT, Hodgkin D, Thomas JP, Ritter G, Chilingerian J. Effect of periodic health exam on provider management of preventive services. $J$ Eval Clin Pract. 2019;25(5):827-833. doi:10.1111/jep.13083

5. Sommer I, Titscher V, Teufer B, et al. Evidence-based recommendations for the revision of the Austrian periodic health examination. Wiener medizinische Wochenschrift. 2019;169(13-14):339-349. doi:10.1007/s10354-019-0699-6

6. Levey AS, Eckardt KU, Tsukamoto Y, et al. Definition and classification of chronic kidney disease: a position statement from Kidney Disease: improving Global Outcomes (KDIGO). Kidney Int. 2005;67(6):2089-2100. doi:10.1111/j.1523-1755.2005.00365.x

7. Danese MD, Powe NR, Sawin CT, Ladenson PW. Screening for mild thyroid failure at the periodic health examination: a decision and cost-effectiveness analysis. JAMA. 1996;276(4):285-292. doi:10.1001/jama.1996.03540040029029

8. Matsuda S. Regulatory effects of health examination programs on medical expenditures for the elderly in Japan. Social Sci Med. 1996;42(5):661-670. doi:10.1016/0277-9536(95)00197-2

9. Wang W, Wu S, Hong Z, et al. Change of incidence of stroke after a community-based intervention for nine years in three cities in China. Chin J Chron Non-commun Dis. 2002;4:30-33.

10. Wang L, Kong L, Wu F, Bai Y, Burton R. Preventing chronic diseases in China. Lancet. 2005;366(9499):1821-1824. doi:10.1016/S01406736(05)67344-8

11. Soriano JB, Zielinski J, Price D. Screening for and early detection of chronic obstructive pulmonary disease. Lancet. 2009;374 (9691):721-732. doi:10.1016/S0140-6736(09)61290-3

12. Fenton JJ, Cai Y, Weiss NS, et al. Delivery of cancer screening: how important is the preventive health examination? Arch Intern Med. 2007;167(6):580-585. doi:10.1001/archinte.167.6.580

13. Vuong Q. Survey data on Vietnamese propensity to attend periodic general health examinations. Scientific data. 2017;4(1):1-10.

14. Liu X, Li N, Liu C, et al. Urban-rural disparity in utilization of preventive care services in China. Medicine. 2016;95(37):e4783. doi:10.1097/MD.0000000000004783

15. World Health Organization. World Report on Ageing and Health[M]. Geneva, Switzerland: Publications of the World Health Organization; 2015.

16. Guo B, Xie X, Wu Q, et al. Inequality in the health services utilization in rural and urban china: a horizontal inequality analysis. Medicine. 2020;99(2):e18625. doi:10.1097/MD.0000000000018625

17. Bluethmann SM, Bartholomew LK, Murphy CC, Vernon SW. Use of Theory in Behavior Change Interventions. Health Educ Behav. 2017;44(2):245-253. doi:10.1177/1090198116647712

18. Samami E, Seyedi-Andi SJ, Bayat B, Shojaeizadeh D, Tori NA. The effect of educational intervention based on the health belief model on knowledge, attitude, and function of women about Pap smear test at Iranian health centers: a randomized controlled clinical trial. $J$ Educ Health Promot. 2021;10(1):22. doi:10.4103/jehp.jehp_33_20

19. Mohamed NC. Validity and reliability of health belief model questionnaire for promoting breast self-examination and screening mammogram for early cancer detection. Scientific data. 2019;20(9):2865.

20. Loke AY, Davies L, Li S. Factors influencing the decision that women make on their mode of delivery: the Health Belief Model. BMC Health Services Res. 2015;15(1):1-12.

21. Namdar A, Bigizadeh S. Measuring Health Belief Model components in adopting preventive behaviors of cervical cancer. J Fasa Univ Med Sci. 2012;2(1):34-44.

22. Conte KP, Schure MB, Goins RTJA. Correlates of social support in older American Indians: the native elder care study. Aging amp. 2015;19(9):835-843. doi:10.1080/13607863.2014.967171

23. Sun X, Chen Y, Tong X, et al. The use of annual physical examinations among the elderly in rural China: a cross-sectional study. BMC Health Serv Res. 2014;14(1):16. doi:10.1186/1472-6963-14-16
24. Ataei P, Gholamrezai S, Movahedi R. An analysis of farmers' intention to use green pesticides: the application of the extended theory of planned behavior and health belief model. $J$ Rural Stud. 2021;81:374-384.

25. Manika D, Golden LL, Brockett PL. H1N1 Prevention Behaviors in Australia: implications from an Extended Health Belief Model. In: The Customer is NOT Always Right? Marketing Orientationsin a Dynamic Business World. Springer; 2017:285-286.

26. Ghaffari M, Gharlipour Z. Related factors of the preventing behaviors of HIV/AIDS among young people: applying the Extended Health Belief Model (EHBM). Int J Pediatrics. 2016;4(8):2317-2328.

27. Buglar ME, White KM, Robinson NG. The role of self-efficacy in dental patients' brushing and flossing: testing an extended Health Belief Model. Patient Educ Couns. 2010;78(2):269-272. doi:10.1016/j.pec.2009.06.014

28. Green EC, Murphy EM. The Health Belief Model. Int $J$ Med. 2020;211-214.

29. Chen Y, Hicks A. Quality of life and related factors: a questionnaire survey of older people living alone in Mainland China. Quality of Life Research: An International Journal of Quality of Life Aspects of Treatment, Care and Rehabilitation. 2014;23(5):1593-1602. doi:10.1007/s11136-013-0587-2

30. Silverstein M, Cong Z, Intergenerational Transfers LS. Living Arrangements of Older People in Rural China: consequences for Psychological Well-Being. J Gerontology. 2006;61(5):S256-S266.

31. Carpenter CJ. A meta-analysis of the effectiveness of health belief model variables in predicting behavior. Health Commun. 2010;25 (8):661-669. doi:10.1080/10410236.2010.521906

32. Janz NK, Becker MH. The Health Belief Model: a decade later. Health Educ Q. 1984;11(1):1-47. doi:10.1177/109019818401100101

33. Harrison JA, Mullen PD, Green LW. A meta-analysis of studies of the Health Belief Model with adults. Health Educ Res. 1992;7 (1):107-116. doi:10.1093/her/7.1.107

34. Rosenstock IM. Why people use health services. Milbank Mem Fund Q. 1966;44(3):94-127. doi:10.2307/3348967

35. Alhalaseh L, Fayoumi H, Khalil B. The Health Belief Model in predicting healthcare workers' intention for influenza vaccine uptake in Jordan. Vaccine. 2020;38(46):7372-7378. doi:10.1016/j. vaccine.2020.09.002

36. Wong LP, Alias H, Wong PF, Lee HY, Abubakar S. The use of the health belief model to assess predictors of intent to receive the COVID-19 vaccine and willingness to pay. Hum Vaccin Immunother. 2020;16(9):2204-2214. doi:10.1080/21645515.2020.1790279

37. Zhao J, Song F, Ren S, et al. Predictors of condom use behaviors based on the Health Belief Model (HBM) among female sex workers: a cross-sectional study in Hubei Province, China. PLoS One. 2012;7 (11):e49542. doi:10.1371/journal.pone.0049542

38. Greenfield SF, Hufford MR, Vagge LM, Muenz LR, Costello ME, Weiss RD. The relationship of self-efficacy expectancies to relapse among alcohol dependent men and women: a prospective study. Journal of Studies on Alcohol. 2000;61(2):345-351. doi:10.15288/ jsa.2000.61.345

39. Jirojwong S, MacLennan R. Health beliefs, perceived self-efficacy, and breast self-examination among Thai migrants in Brisbane. Journal of Advanced Nursing. 2003;41(3):241-249. doi:10.1046/ j.1365-2648.2003.02552.x

40. Isa A, Muhamad NA, Mustapha N, Mutalip MHA, Royali MSM, Baharin MF. Association between self-efficacy and health behaviour in disease control: a systematic review. Int J Pediatrics. 2018;10 (1):1-18.

41. Farmer D, Reddick B, D’Agostino R, Jackson SA. Psychosocial correlates of mammography screening in older African American women. Oncol Nurs Forum. 2007;34(1):117-123. doi:10.1188/07. ONF.117-123 
42. Lai DW, Kalyniak S. Use of annual physical examinations by aging Chinese Canadians. J Aging Health. 2005;17(5):573-591. doi:10.1177/0898264305279778

43. Mitsuhashi Y, Kishi R, Ecuchi T, et al. [Study of influences of social support and social networks on participation in periodic health examination of the elderly at home comparison of three regions with different social backgrounds]. Japanese $j$ Public Health. 2006;53 (2):92-104.

44. Feng GC, Lin Z, Ou W, Su X, Yan Q. A Model-Based Meta-Analysis of Willingness to Participate in Cancer Screening. Int J Environ Res Public Health. 2021;18(5):5. doi:10.3390/ijerph18052580

45. Loke AY, Davies L, Li SF. Factors influencing the decision that women make on their mode of delivery: the Health Belief Model. BMC Health Serv Res. 2015;15:274. doi:10.1186/s12913-015-0931-z

46. Dündar PE, Ozmen D, Oztürk B, et al. The knowledge and attitudes of breast self-examination and mammography in a group of women in a rural area in western Turkey. BMC Cancer. 2006;6:43. doi:10.1186/1471-2407-6-43

47. Green CA, Pope CR. Gender, psychosocial factors and the use of medical services: a longitudinal analysis. Social Sci Med. 1999;48 (10):1363-1372. doi:10.1016/S0277-9536(98)00440-7

48. Dodel M. Cyber-victimization preventive behavior: a health belief model approach. Computers in Human behav. 2017;68:359-367.
49. Didarloo A, Nabilou B. Psychosocial predictors of breast self-examination behavior among female students: an application of the health belief model using logistic regression. Int J Med. 2017;17 (1):1-8.

50. Redondo-Sendino A, Guallar-Castillón P, Banegas JR, RodríguezArtalejo F. Gender differences in the utilization of health-care services among the older adult population of Spain. BMC Public Health. 2006;6:155. doi:10.1186/1471-2458-6-155

51. Hammond WP, Matthews D, Corbie-Smith G. Psychosocial factors associated with routine health examination scheduling and receipt among African American men. J Natl Med Assoc. 2010;102 (4):276-289. doi:10.1016/S0027-9684(15)30600-3

52. Almasi A, Mohammadi M, Azizi A, et al. Conservation, Recycling: assessing the knowledge, attitude and practice of the kermanshahi women towards reducing, recycling and reusing of municipal solid waste. Resources Conservation Recycl. 2019;141:329-338. doi:10.1016/j.radonc.2019.07.019

53. Lee MC. Knowledge, barriers, and motivators related to cervical cancer screening among Korean-American women. A Focus Group Approach Cancer Nursing. 2000;23(3):168-175.
Patient Preference and Adherence

\section{Publish your work in this journal}

Patient Preference and Adherence is an international, peer-reviewed, open access journal that focusing on the growing importance of patient preference and adherence throughout the therapeutic continuum. Patient satisfaction, acceptability, quality of life, compliance, persistence and their role in developing new therapeutic modalities and compounds to optimize clinical outcomes for existing disease
Dovepress

states are major areas of interest for the journal. This journal has been accepted for indexing on PubMed Central. The manuscript management system is completely online and includes a very quick and fair peer-review system, which is all easy to use. Visit http:// www.dovepress.com/testimonials.php to read real quotes from published authors. 